\title{
CORRIGENDUM
}

\section{A model of tear-film breakup with continuous mucin concentration and viscosity profiles - CORRIGENDUM}

\author{
Mohar Dey, Atul S. Vivek, Harish N. Dixit, Ashutosh Richhariya \\ and James J. Feng
}

doi:10.1017/jfm.2018.776, Published by Cambridge University Press,

6 November 2018

Two errors were made in evaluating the dimensional parameters of table 1 and in converting them to the dimensionless parameters in Dey et al. (2019). This led to an incorrect time scale in computing the dimensional tear-film breakup time in figure 12 . All the other results, presented in figures 3-11, are dimensionless and remain correct for the dimensionless parameters employed therein.

To rectify the errors, we first reinstate a factor of $6 \pi$ in the Hamaker constant omitted by mistake; the correct value is $\mathcal{A}=6 \pi \times 3.5 \times 10^{-19} \mathrm{~J} \mathrm{~m}$ (Braun et al. 2018). Besides, we revise the tear-film thickness to $H=0.5 \mu \mathrm{m}$. The new $H$ value is thinner than the experimentally reported tear-film thickness by a factor of 7-10. This is based on recent findings that the tear film undergoes a rapid initial thinning owing to factors such as evaporation (Braun et al. 2018), lipid clustering on the interface (Zhong et al. 2019) and lid-associated thinning, including drainage (King-Smith, Begley \& Braun 2018). Thus, within several seconds, the tear film thins from a thickness of about $3.5 \mu \mathrm{m}$ to $0.5 \mu \mathrm{m}$ (Braun et al. 2018). It is only after this initial phase that the van der Waals force becomes the dominant driving force for tear-film rupture. As our continuous viscosity model $(\mathrm{CVM})$ focuses solely on the van der Waals-driven rupture and ignores the initial rapid thinning, we should adopt the $H$ value at the end of the rapid thinning. The changes in $\mathcal{A}$ and $H$ lead to new values for four dimensionless parameters: $\mathcal{C}=0.03, P e_{s}=0.02, \Delta_{b}=10^{-4}$ and $\mathcal{M}=0.05$. With the corrected parameters and time scale, figure 1 plots the dimensional rupture time $t_{\text {rup }}$ as a function of $\mathcal{C}$. This replaces the original figure 12 .

The new $t_{\text {rup }}$ turns out to be quite close to that reported in the original figure 12 . For a reasonable range of the interfacial tension, $\mathcal{C}$ now lies in the range of $10^{-3}-0.1$, over which $t_{\text {rup }}$ varies from 2.4 to $135 \mathrm{~s}$. For the baseline value $\mathcal{C}=0.03$, the rupture time is $t_{\text {rup }}=44.4 \mathrm{~s}$. Hence, the previous conclusion - drawn from comparisons with experimental data and two-layer model predictions - stands: the CVM is superior in reproducing experimental measurements of healthy-eye tear film breakup time.

\section{Acknowledgement}

The correction of the errors was largely done by A. Choudhury of the Indian Institute of Technology - Hyderabad during a research visit at the University of British Columbia, Vancouver. 


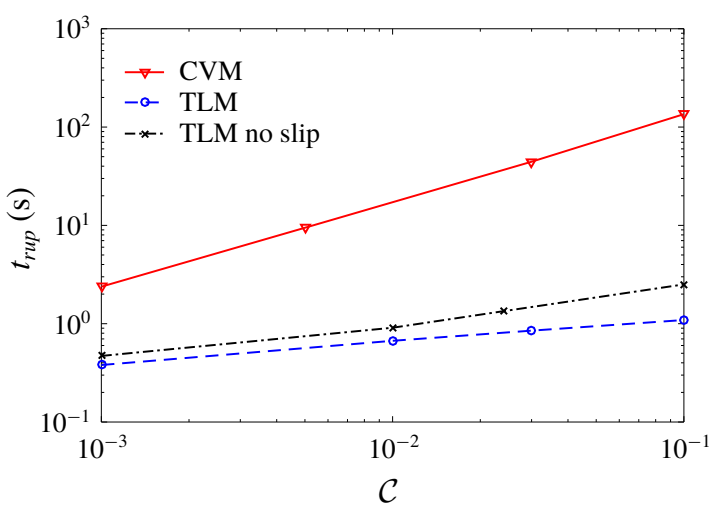

FIgURE 1. Dimensional rupture time $t_{\text {rup }}$ as a function of the dimensionless surface tension $\mathcal{C}$. The dotted lines are predictions of the two-layer model (TLM) with and without slip on the substrate. This replaces the original figure 12 in Dey et al. (2019).

\section{Declaration of interests}

The authors report no conflict of interest.

\section{REFERENCES}

Braun, R. J., Driscoll, T. A., Begley, C. G., King-Smith, P. E. \& Siddique, J. I. 2018 On tear film breakup (TBU): dynamics and imaging. Math. Med. Biol. 35, 145-180.

Dey, M., Vivek, A. S., Dixit, H. N., Richhariya, A. \& Feng, J. J. 2019 A model of tear-film breakup with continuous mucin concentration and viscosity profiles. J. Fluid Mech. 858, 352-376.

King-Smith, P. E., Begley, C. G. \& Braun, R. J. 2018 Mechanisms, imaging and structure of tear film breakup. Ocul. Surf. 16, 4-30.

Zhong, L., Ketelaar, C. F., Braun, R. J., Begley, C. G. \& King-Smith, P. E. 2019 Mathematical modelling of glob-driven tear film breakup. Math. Med. Biol. 36, 55-91. 